\title{
Relationship between the neutron time delay distribution and the rigidity spectrum of primary cosmic rays up to $16.8 \mathrm{GV}$
}

\author{
P.-S. Mangeard ${ }^{* a, b}$ D. Ruffolo, ${ }^{b, c}$ A. Sáiz, ${ }^{b, c}$ W. Nuntiyakul, ${ }^{b, c, d}$ S. Madlee, ${ }^{b}$ T. \\ Nutaro $, c, e$ J. W. Bieber, ${ }^{f}$ J. Clem, ${ }^{f}$ P. Evenson, ${ }^{f}$ R. Pyle, ${ }^{f}$ M. L. Duldig, ${ }^{g}$ J. E. Humble ${ }^{g}$ \\ ${ }^{a}$ National Astronomical Research Institute of Thailand (NARIT), Chiang Mai 50200, Thailand. \\ ${ }^{b}$ Department of Physics, Faculty of Science, Mahidol University, Bangkok 10400, Thailand. \\ ${ }^{c}$ Thailand Center of Excellence in Physics, CHE, Ministry of Education, Bangkok 10400, \\ Thailand. \\ ${ }^{d}$ Faculty of Science, Chandrakasem Rajabhat University, Bangkok 10900, Thailand. \\ ${ }^{e}$ Department of Physics, Faculty of Science, Ubon Ratchathani University, Ubon Ratchathani \\ 34190, Thailand. \\ ${ }^{f}$ Bartol Research Institute, University of Delaware, Newark, DE 19716, USA. \\ ${ }^{g}$ School of Physical Sciences, University of Tasmania, Hobart, Tasmania 7001, Australia. \\ E-mail: psmangeardegmail.com, david.rufemahidol.ac.th, \\ alejandro.sai@mahidol.ac.th, w.nuntiyakul@gmail.com, \\ suttiwat.mademahidol.ac.th,tnutaro@yahoo.com, \\ jwbieber@bartol. udel.edu, clem@bartol. udel.edu, \\ evenson@bartol. udel.edu, rogerpyle@gmail.com, \\ marc.duldig@utas.edu.au, john.humble@utas.edu.au.
}

Neutron monitors are the premier instruments for precisely tracking time variations in the Galactic cosmic ray flux at GeV-range energies above the geomagnetic cutoff at the location of measurement. In addition to the count rate, recording and analyzing the time delays between successive counts allows us to infer variations in the cosmic ray spectrum as well. In particular, we can determine the leader fraction $L$, defined as the fraction of neutrons that did not follow a previous neutron detection in the same tube from the same nuclear interaction, from time delay histograms. By analyzing data taken during 2001-2007 by a ship-borne neutron monitor latitude survey we confirm a strong dependence of $L$ on the geomagnetic cutoff up to $16 \mathrm{GV}$. The data of the Princess Sirindhorn Neutron Monitor located in Thailand at a higher vertical cutoff of $16.8 \mathrm{GV}$ have also been analyzed for the period 2007-2014. We have developed Monte Carlo simulations of cosmic ray interactions in the atmosphere and in both neutron monitors. Both data and simulations show that the absolute value of $L$ depends significantly of the configuration of the detector and that experimental conditions such as the electronic dead time must be well monitored. The simulation results show a change in $L$ with the geomagnetic cutoff as observed by the latitude survey, confirming that this change in $L$ can reasonably be attributed to changes in the cosmic ray spectrum.

The 34th International Cosmic Ray Conference,

30 July- 6 August, 2015

The Hague, The Netherlands

\footnotetext{
* Speaker.
} 


\section{Introduction}

Neutron monitors (NMs) are widely used ground-based detectors of the secondary particles induced by the interactions of cosmic rays in the Earth's atmosphere. They consist of several proportional counters which are sensitive to the neutrons produced by the interaction of the secondary particles (mostly neutrons) with a dense producer, typically $\mathrm{Pb}$. The NMs provide accurate hourly count rates and can track the variations of the galactic cosmic ray (GCR) flux, in particular the long term modulation (11 and 22 year cycles) from solar activity as well as the short term variations, e.g., the Forbush decreases that may follow solar storms. Combining data from multiple NMs can in theory indicate variations of the cosmic ray spectrum. However, such analysis requires an inter-calibration to $\sim 0.2 \%$ of the yield functions of different detectors and achieving this accuracy proves to be very challenging both experimentally $[3,10]$ and computationally via Monte Carlo simulation [7]. Recording and analyzing the time between successive counts may allow us to track the variations of the secondary particle spectrum using a single neutron monitor, thus avoiding such systematic uncertainties. Recording the time delays between counts [4] allows us to evaluate two parameters that can depend on the GCR spectrum: $i$ ) the multiplicity $M$ which corresponds to the number of pulses or counts within a time window (typically a few ms long) and ii) the leader fraction $L$ which is the fraction of neutron counts not associated with a previous neutron count in the same tube from the same $\mathrm{Pb}$ nuclear interaction. Note that $M$ is contaminated by chance coincidences but $L$ is not. It is possible to assess a non-contaminated, or pure, multiplicity $M_{\mathrm{p}}=1 / L$.

We analyzed the time delay distributions of a ship-borne neutron monitor (standard "3NM64") and extracted the leader fraction for six latitude surveys from 2001 to 2007 . We report in this work the dependence of $L$ on the effective apparent cutoff rigidity [6] up to $16 \mathrm{GV}$ and with the solar modulation during that period. We compare the results with our Monte Carlo simulation of the interaction of the cosmic rays in the atmosphere and of the secondary particles in the 3NM64 within a simple model of its surroundings. We extended the results at an higher apparent cutoff rigidity of $17.3 \mathrm{GV}$ by analyzing the time delay distributions recorded by the Princess Sirindhorn Neutron Monitor (PSNM, standard "18NM64") since it was installed in 2007. With the help of a very detailed simulation of the PSNM response, we report a strong dependence of the leader fraction on the configuration of the detector, the local environment and the electronic dead time.

\section{Extraction of the leader fraction from the time delay distribution}

The time delay histograms (Figure 1) contain both chance coincidence and nuclear physics contributions from the interaction of the neutron with a $\mathrm{Pb}$ nucleus. The long time delays are dominated by chance coincidences whereas short time delays are dominated by counts of neutrons that originate from the same $\mathrm{Pb}$ nucleus when hit by a secondary particle. Note here that the two contributions are not independent. It is possible to extract the parameter $L$ from the long time delay histogram via the following statistical analysis. For every hour and for each counter independently, the histograms are filled with the time between successive counts. Consequently, each histogram can be normalized to obtain a probability density function $n(t)$, where, $n(t) \mathrm{d} t$ represents the probability of having another neutron count after a time delay between $t$ and $t+\mathrm{d} t$. We are in presence of a non-homogeneous Poisson process so if we define $R(t)$ as the probability 

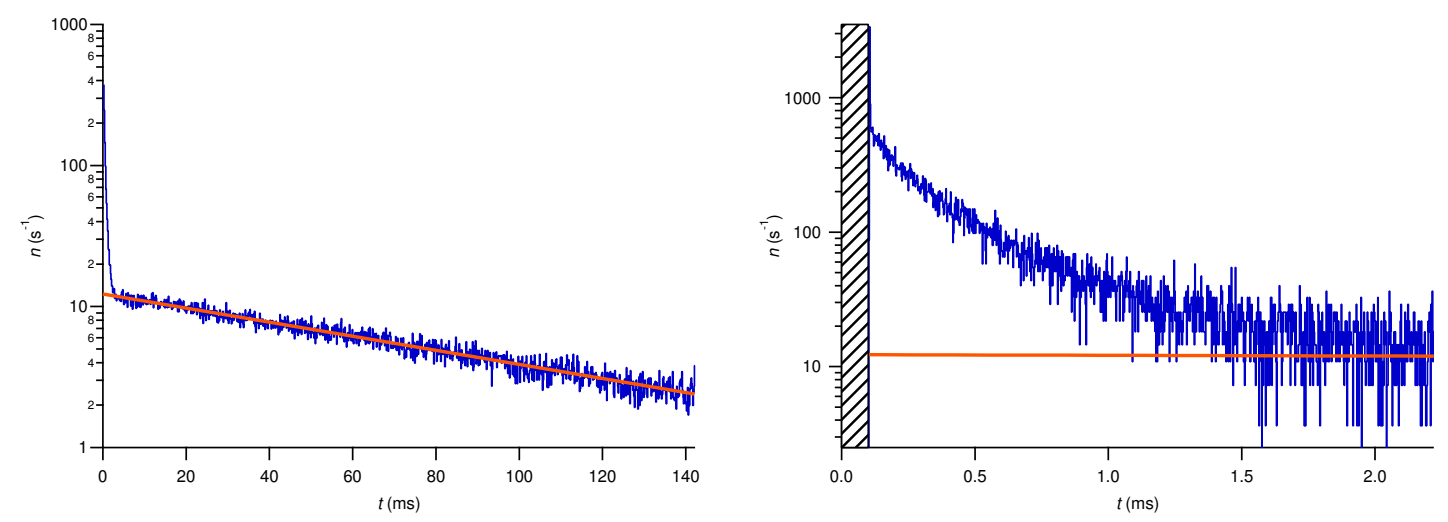

Figure 1: Example of the time delay distribution at one counter tube in the ship-borne neutron monitor, recorded during three hours. Long time delays (left) show the exponential distribution typical of unrelated events, while short time delays (right) deviate substantially from the exponential function (red line).

that a neutron count in one counter tube is the first recorded count within a time delay $t$,

$$
\frac{\mathrm{d} R}{\mathrm{~d} t}(t)=-(\alpha+\beta(t)) R(t) \quad \text { and } \quad R\left(t_{\mathrm{d}}\right)=1,
$$

where $\alpha$ and $\beta(t)$ are respectively the probability of a chance coincidence per unit of time and the probability of detecting a follower count per unit of time. Here $t_{\mathrm{d}}$ is the electronic data acquisition system dead time. Let $R_{\mathrm{n}}(t)$ be the purely nuclear contribution to $R$ so that:

$$
R(t)=\mathrm{e}^{-\alpha\left(t-t_{\mathrm{d}}\right)} R_{\mathrm{n}}(t) \quad \text { with } \quad R_{\mathrm{n}}(t) \equiv \mathrm{e}^{-\int_{t_{\mathrm{d}}}^{t} \beta\left(t^{\prime}\right) \mathrm{d} t^{\prime}} .
$$

We can relate $n(t)$ with $R(t)$ via:

$$
\begin{gathered}
R(t) \equiv 1-\int_{t_{\mathrm{d}}}^{t} n\left(t^{\prime}\right) d t^{\prime} \quad \text { or } \quad n(t)=-\frac{\mathrm{d} R}{\mathrm{~d} t}(t), \\
n(t)=\alpha \mathrm{e}^{-\alpha\left(t-t_{\mathrm{d}}\right)} R_{\mathrm{n}}(t)-\mathrm{e}^{-\alpha\left(t-t_{\mathrm{d}}\right)} \frac{\mathrm{d} R_{\mathrm{n}}}{\mathrm{d} t}(t) .
\end{gathered}
$$

For $t>5 \mathrm{~ms}$, the nuclear contribution tends to be negligible so the probability to have a follower tends to zero, i.e., we have $\beta(t ; t>5 \mathrm{~ms}) \rightarrow 0$ which implies that $R_{\mathrm{n}}(t ; t>5 \mathrm{~ms}) \rightarrow L$. Indeed, at large time delays, $R_{\mathrm{n}}(t)$ represents the probability that a count did not follow a previous count from the same $\mathrm{Pb}$ interaction in the same counter which is the definition of the leader fraction. For $t>5 \mathrm{~ms}$, we have:

$$
n(t) \simeq \alpha L \mathrm{e}^{-\alpha\left(t-t_{\mathrm{d}}\right)} .
$$

Due to an overflow every $t_{0}=142 \mathrm{~ms}$ in the electronic time delay variable, the long time delay histogram actually records:

$$
\tilde{n}(t)=\alpha L \sum_{k=0}^{\infty} \mathrm{e}^{-\alpha\left(t+k t_{0}-t_{\mathrm{d}}\right)}=\frac{\alpha L \mathrm{e}^{\alpha t_{\mathrm{d}}}}{1-\mathrm{e}^{-\alpha t_{0}}} \mathrm{e}^{-\alpha t} .
$$

$L$ can be calculated as:

$$
L=\frac{1-\mathrm{e}^{-\alpha t_{0}}}{\alpha \mathrm{e}^{\alpha t_{\mathrm{d}}}} A
$$

where $A$ and $\alpha$ are the parameters from the histogram fitted with $\tilde{n}(t)=A \mathrm{e}^{-\alpha t}$ at long $t$. 

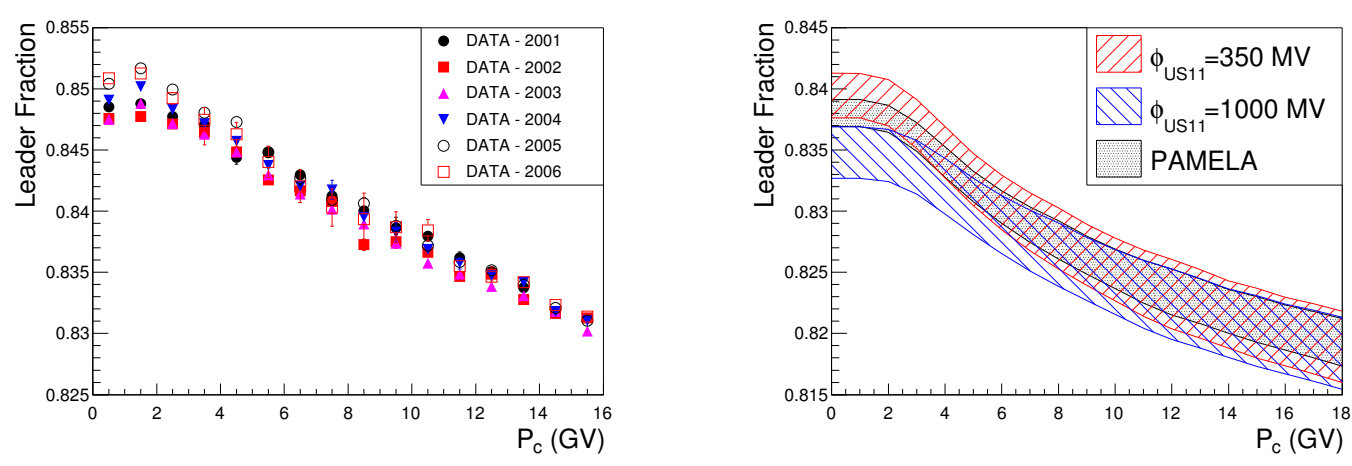

Figure 2: (Left) Dependence of the leader fraction $L$ on the apparent cutoff rigidity $P_{\mathrm{c}}$ for the six surveys. Each point shows the mean value of $L$ for the corresponding bin and the error bar corresponds to the standard error. (Right) Dependence of the simulated leader fraction on the apparent cutoff rigidity $P_{\mathrm{c}}$ for two values of the solar modulation intensity that bracket the range of values of $\phi_{U S 11}$ from [13] during the latitude survey period. The bands show the range of values for several assumptions of local interstellar spectrum from [9] and for five different profiles of atmosphere. PAMELA spectra used here corresponds to a solar modulation of about $400 \mathrm{MV}$ and the band shows the effect from the atmospheric profile.

\section{Sensitivity to the primary cosmic ray spectrum}

The mobile monitor 3NM64 operated in an insulated container on board an icebreaker during several winters from 1994 to 2007 [11]. The typical journey of the ship started from Seattle (USA) in November, traveled to the McMurdo base in Antarctica and returned to Seattle in April. The survey year refers here to the year in which the survey began. During short periods of time, it traveled over a large range of rigidity cutoff, up to $16 \mathrm{GV}$ for the surveys when the time delay histograms were available. They gave a perfect opportunity to study the sensitivity of the leader fraction to the variation of the GCR spectrum. Due to a low cont rate $(4-13 \mathrm{~Hz})$, we merged the histograms over 3-hour periods. We determined the total leader fraction

$$
L=\sum_{i=1}^{3} f_{i} L_{i}
$$

where $L_{i}$ is the leader fraction of the $i^{\text {th }}$ tube calculated from the time delay distribution and $f_{i}$ is the contribution fraction of the $i^{\text {th }}$ tube to the total recorded time delays. $L$ was sorted per bin of cutoff rigidity $P_{\mathrm{c}}$ and the results are presented in Figure 2. First, independently of the survey, a decrease of a few percent from $L \sim 0.85$ to $L \sim 0.83$ is clearly visible when $P_{\mathrm{c}}$ increases within the available range. We can also observed that the solar modulation impacts the value of $L$ in the same way. At low $P_{\mathrm{c}}$, there is a variation up to $\sim 0.004$ over the 6 years with a larger value for survey years 2005 and 2006 with low solar activity. This confirms that $L$ is related to the rigidity spectrum of primary cosmic rays impinging on Earth's atmosphere.

We performed Monte Carlo simulations using the FLUKA 2011 software [5, 8] with the interaction model DPMJET-2.5 as recommended in the FLUKA manual. The method was composed of two stages: $i$ ) the development of the air shower induced by the primary cosmic rays was simulated 
and the secondary particle (SP) fluxes were extracted at the altitude of the detector, and ii) the detector sensitivity within its surroundings was determined for nine types of SPs (protons, neutrons, $\mu^{+}, \mu^{-}, \pi^{+}, \pi^{-}, e^{+}, e^{-}$and $\gamma$ ). We used their outputs to determine a simulated leader fraction. The atmospheric profiles combined information from two models: the Global Data Assimilation System (GDAS) at low altitude, and the Naval Research Laboratory Mass Spectrometer, Incoherent Scatter Radar Extended model (NRLMSISE-00, [12]) for dry air at higher altitude up to $72.5 \mathrm{~km}$. To estimate the effect of atmospheric structure, we computed the first stage of the simulation for several types of atmosphere corresponding to several locations encountered by the ship (Seattle, Honolulu, Hobart and McMurdo). The flux of cosmic rays was assumed isotropic and an apparent geomagnetic cutoff was applied. The systematic effects from the choice of the primary cosmic ray spectrum $[1,2,9]$ and from solar modulation were also estimated. The simulated results are shown in Figure 2. $L_{\mathrm{MC}}$ is underestimated by less than $2 \%$, which may be explained by a too simple model of the ship structure in the simulation. In terms of relative variation, $L_{\mathrm{MC}}$ decreases by $\sim 2 \%$ with the rigidity cutoff in the range of interest. The variation of $L_{\mathrm{MC}}$ with the solar modulation is clearly visible. The overlap of the bands shows that the variation with solar modulation is smaller when $P_{\mathrm{c}}$ increases, as in the observation. This confirms that $L$ can be used as an indicator of spectral changes in the primary cosmic ray flux at the top of the atmosphere.

\section{Dependence on local environment and instrumental details}

In order to study the spectral information contained in $L$, it is crucial to eliminate the effects of the local environment from the data. The Princess Sirindhorn neutron monitor (PSNM), an 18NM64 detector located at Doi Inthanon, Thailand's highest mountain (2565 m o.s.1.), can be very useful at this point to gain insight on local (atmospheric) effects, thanks to its high count rate and relatively stable configuration. We analyzed more than 6 years of data (December 2007February 2014) taken at PSNM by extracting $L$ for each of the 18 counter tubes from their hourly or daily time delay histograms. A clear 1-year oscillation appears in the data, indicating that this "uncorrected" value of $L$ is dominated by seasonal effects. Variables such as the atmospheric depth, amount of water in the atmosphere, temperature, cloud cover, or rain, which are themselves correlated or anticorrelated to one another, may induce variations in our measurement of $L$.

The atmospheric depth, for which the atmospheric pressure $P$ is a good proxy, changes seasonally, and also has characteristic $24-\mathrm{h}$ and 12 -h oscillations due to atmospheric tides. Therefore we can use these short-term variations, typically not present in other environmental variables, to characterize and remove the effect of atmospheric depth. We perform this so-called "pressure correction" by fitting the scatter plots of $\Delta \log L_{j}$ versus $\Delta P$ for each tube $j$ to straight lines of slope $b_{j}$. Here $\Delta$ denotes the residual with respect to a 24-h moving average. The (positive) correlation in these plots is small but significant. We find values for $b_{j}$ to be in the range $0.015-0.023 \% \mathrm{mmHg}^{-1}$. The values of $L_{j}$ can then be multiplied by $\exp \left(-b_{j}\left(P-P_{0}\right)\right)$ to eliminate the pressure dependence.

To correct for other environmental effects we use the atmospheric water vapor partial pressure $E_{\mathrm{w}}$ (estimated from GDAS at the location of Doi Inthanon) as a general proxy. The correlation between $L$ and $E_{\mathrm{w}}$ is negligible at low $E_{\mathrm{w}}$ but becomes stronger at higher $E_{\mathrm{w}}$. To model this dependence, we fit the scatter plots of pressure-corrected $L_{j}$ vs $E_{\mathrm{w}}$ to a power law such that

$$
L_{j}\left(E_{\mathrm{w}}\right) \sim L_{j}\left(E_{\mathrm{w}, 0}\right)\left[1-k_{j}\left(E_{\mathrm{w}}^{\gamma_{j}}-E_{\mathrm{w}, 0}^{\gamma_{j}}\right) /(12 \mathrm{mmHg})^{\gamma_{j}}\right] .
$$




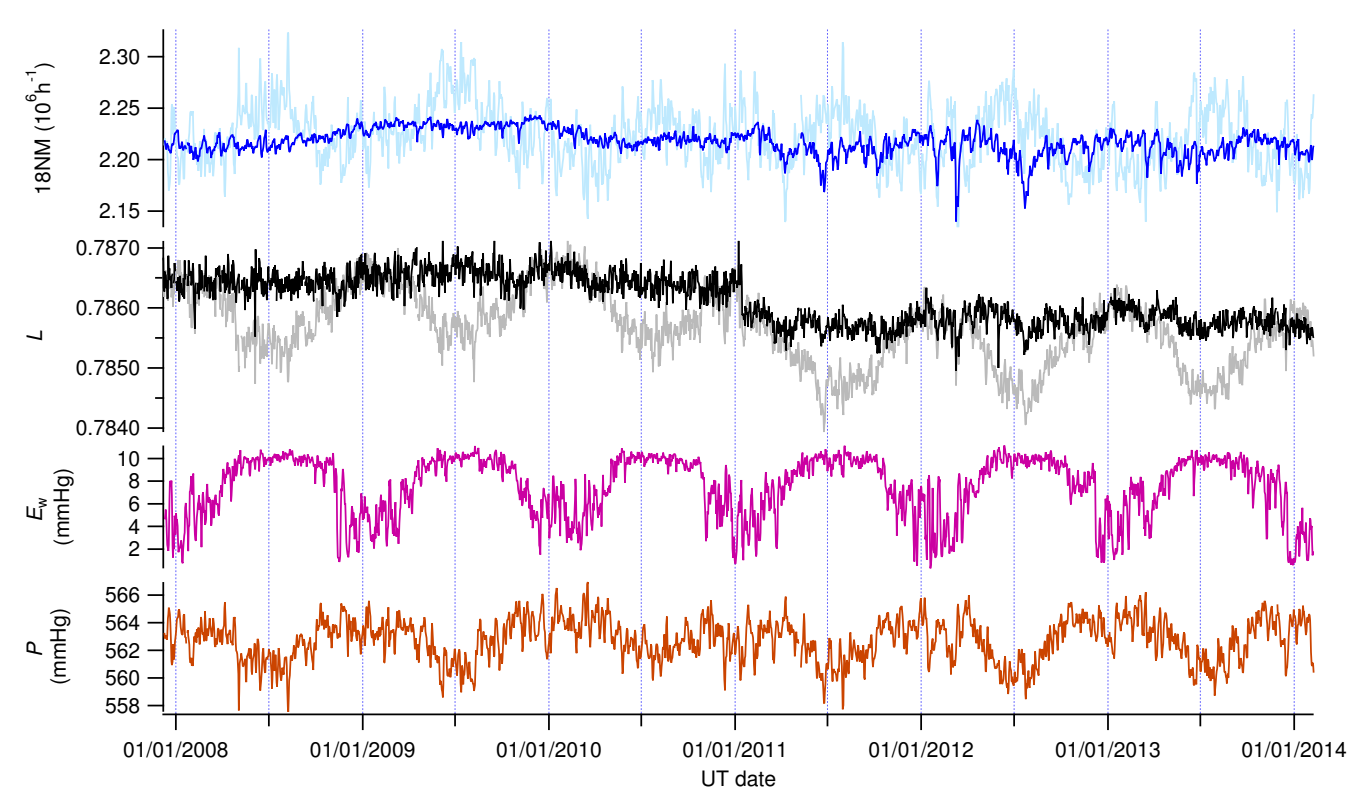

Figure 3: From top to bottom:. 18NM, PSNM total (18 tubes) count rate, pressure corrected (dark blue) and uncorrected (light blue); $L$, leader fraction calculated from the 18 tubes, corrected for atmospheric effects (black) and uncorrected (grey) (the shift in January 2011 is due to an upgrade of the electronics); $E_{\mathrm{w}}$, water vapor pressure at the $750 \mathrm{hPa}$ layer from the GDAS; $P$, atmospheric pressure measured at the station.

We find $k_{j} \sim 0.001-0.002$, and $\gamma_{j} \sim 1.1-3.0$. Each $L_{j}$ can then be "vapor corrected" by dividing it with the term in square brackets in (4.1). The resultant values of $L_{j}$ after pressure and vapor corrections have no measurable correlation with environmental factors and are assumed to depend mostly on extraterrestrial factors. The average $L$ of the 18 individual $L_{j}$ 's studied here is shown in Figure 3 both before and after a correction to $P_{0}=563.4 \mathrm{mmHg}$ and $E_{\mathrm{w}, 0}=4.7 \mathrm{mmHg}$. The observed variations in $L$, appearing both as long-term trends and short-term features such as Forbush decreases, roughly follow the variations in count rate. This agrees with the fact that space physics processes that reduce the flux of cosmic rays generally do so more at lower energies than at higher energies, thus increasing the relative abundance of higher energy atmospheric particles that hit the detector and therefore the fraction of follower counts. This indicates that $L$ can also be used to track short-term spectral changes using data at a single location.

On the other hand, to compare measurements of $L$ for different detectors it must be noticed that the instrument details can also influence the exact value of $L$. Most systems have a dead time $t_{\mathrm{d}}$ after each count during which it is not possible to register a new count. Due to the sharp peak at small $t$ in the time delay histogram (Figure 1), the exact value of $t_{\mathrm{d}}$ controls the measured $L$. This does not normally affect the study of $L$ variations, since $t_{\mathrm{d}}$ is normally stable for a given system. Figure 4 shows the effect of $t_{\mathrm{d}}$ on $L_{j}$ for each of the 18 neutron counters of the PSNM. Time delay histograms are recorded with a nominal $t_{\mathrm{d}} \sim 80 \mu \mathrm{s}$, but six of the counters have $t_{\mathrm{d}} \sim 88 \mu \mathrm{s}$ due to a difference in one of the electronic components. This clearly affects the value of $L_{j}$. Another significant effect is the position of the counter in the detector. Tubes near the edges are surrounded by a larger amount of reflector, which brings back some produced neutrons that otherwise would 

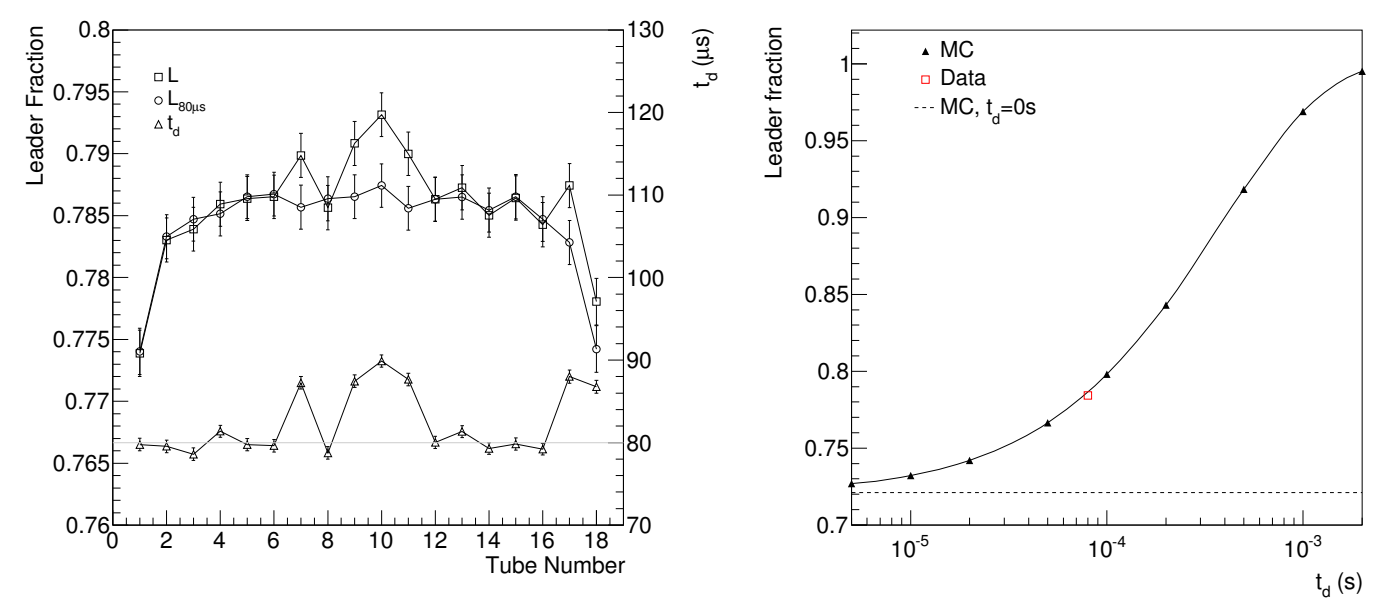

Figure 4: (Left) Squares: means of $L_{j}$ hourly values as a function of tube number $j=1, \ldots, 18$ during the 3-year interval Jan. 2011-Feb. 2014. Error bars denote standard deviations. Circles: $L_{j}$ values estimated for a dead time $t_{\mathrm{d}}=80 \mu \mathrm{s}$. Triangles: measured values of $t_{\mathrm{d}}$. (Right) Average $L$ at different values of $t_{\mathrm{d}}$ for a MC model of the PSNM that uses an atmospheric profile for Doi Inthanon (based on GDAS and NRLMSISE-00) with $P=563.4 \mathrm{mmHg}$ and $E_{\mathrm{w}}=4.7 \mathrm{mmHg}$. The square is the average of the 18 circles in the left panel. The horizontal line shows the modeled no-dead-time $\left(t_{\mathrm{d}}=0\right)$ value of $L$.

give a count in another tube, thus decreasing $L_{j}$. Figure 4 also shows the results of MC simulations of the PSNM that we used to study the dependence of $L$ on $t_{\mathrm{d}}$. In the simulation, all the tubes are modeled with the same $t_{\mathrm{d}}$. The modeled average of $L$ for the 18 tubes agrees with the observed value within $0.5 \%$. This analysis also suggests that a small value of the instrument dead time is crucial for its sensitivity to spectral variations in the GCR.

\section{Conclusions}

We studied the time delays between successive counts in the same counter of a neutron monitor. We developed a statistical method to extract a single parameter, the leader fraction $L$, from the recorded time delay distributions. By analyzing the data from a mobile monitor during several latitude surveys and the PSNM, we showed that the variations of $L$ can be used as an indicator of variations in the GCR spectrum. Both Monte Carlo simulations and observations show a strong dependence on pressure and water vapor pressure. In addition to the environmental dependences, the intrinsic characteristics of the detector and the electronic dead time have strong effects on the absolute value of the leader fraction. $L$ provides the capability to assess variation of the primary cosmic ray spectrum with a single neutron monitor.

\section{Acknowledgments}

We thank the Royal Thai Air Force and Chatchai Injai for their kind assistance with the observations. P.-S. M. was partially supported under the postdoctoral research sponsorship of Mahi- 
dol University. This work was supported by the Thailand Research Fund, the United States National Science Foundation (OPP-0838839, PLR-1245939, and their predecessors) and the Australian Antarctic Division.

\section{References}

[1] O. Adriani et al., PAMELA measurements of cosmic-ray proton and helium spectra, Science 332, 69, doi: 10.1126/science.1199172 (2011).

[2] O. Adriani et al., Time dependence of the proton flux measured by PAMELA during the 2006 July-2009 December solar minimum, Astrophys. J., 765:91 (8pp), doi:10.1088/0004-637X/765/2/91 (2013).

[3] N. Aiemsa-ad et al., Measurement and simulation of neutron monitor count rate dependence on surrounding structure, J. Geophys. Res. Space Physics, 120, doi: 10.1002/2015JA021249 (2015).

[4] J. W. Bieber, J. M. Clem, M. L. Duldig, P. A. Evenson, J. E. Humble, and R. Pyle, Latitude survey observations of neutron monitor multiplicity, J. Geophys. Res., 109, A12106, doi:10.1029/2004JA010493 (2004).

[5] T. T. Bohlen, F. Cerutti, M. P. W. Chin, A. Fassò, A. Ferrari, P. G. Ortega, A. Mairani, P. R. Sala, G. Smirnov, and V. Vlachoudis, The FLUKA Code: developments and challenges for high energy and medical applications, Nuclear Data Sheets, 120, 211-214 (2014).

[6] J. Clem, J. W. Bieber, P. Evenson, D. Hall, J. E. Humble, and M. Duldig, Contribution of obliquely incident particles to neutron monitor counting rate, J. Geophys. Res., 102(A12), 26919-26926, doi:10.1029/97JA02366 (1997).

[7] J. Clem and L. Dorman, Neutron monitor response functions, Space Sci. Rev., 93, 335 (2000).

[8] A. Ferrari, P. R. Sala, A. Fassò \& J. Ranft, FLUKA: a multi-particle transport code, CERN-2005-10, INFN/TC_05/11, SLAC-R-773 (2005).

[9] K. Herbst et al. , On the importance of the local interstellar spectrum for the solar modulation parameter, J. Geophys. Res., 115, D00I20, doi:10.1029/2009JD012557 (2010).

[10] H. Krüger, H. Moraal, J. W. Bieber, J. M. Clem, P. A. Evenson, K. R. Pyle, M. L. Duldig, and J. E. Humble, A calibration neutron monitor: Energy response and instrumental temperature sensitivity, $J$. Geophys. Res., 113, A08101, doi:10.1029/2008JA013229 (2008).

[11] W. Nuntiyakul et al., Latitude survey investigation of Galactic cosmic ray solar modulation during 1994-2007, Astrophys. J., 795:11 (13pp), 2014 November 1.

[12] J. M. Picone, A. E. Hedin, D. P. Drob, A. C. Aikin, NRLMSISE-00 empirical model of the atmosphere: Statistical comparisons and scientific issues, J. Geophys. Res., 107, 1468, doi:10.1029/2002JA009430 (2002).

[13] I. G. Usoskin, G. A. Bazilevskaya \& G. A. Kovaltsov, Solar modulation parameter for cosmic rays since 1936 reconstructed from ground-based neutron monitors and ionization, J. Geophys. Res., 116, A02104, doi:10.1029/2010JA016105 (2011). 